\title{
DEPOSICIÓN CONVECTIVA RÁPIDA A ESCALA NANOMÉTRICA DE MATERIALES COMPUESTOS ACTIVOS PARA LA FABRICACIÓN DE TRANSISTORES ORGÁNICOS DE EFECTO DE CAMPO
}

\author{
Rapid Convective Deposition AT \\ Nanoscale of Active Composite \\ MATERIALS FOR THE MANUFACTURE OF \\ ORGANIC FIELD-EFFECT TRANSISTORS
}

\author{
Freddy G. del Pozo ${ }^{1, *}$ (D), Marta Mas-Torrent ${ }^{2}$ (D)
}

Recibido: 24-07-2020, Recibido tras revisión: 04-12-2020, Aceptado: 03-02-2021, Publicado: 01-07-2021

\section{Resumen}

Transistores orgánicos de efecto de campo basados en un material compuesto han sido fabricados por medio de la técnica de deposición convectiva rápida. La fabricación fue llevada a cabo bajo condiciones ambientales (aire, luz y humedad). En todos los casos, los transistores fabricados muestran un claro comportamiento de efecto de campo con características de semiconductor tipo-p, y exhiben movilidades en el orden de $10^{-2} \mathrm{~cm}^{2} /$ V.s, totalmente comparables con transistores obtenidos por evaporación térmica del mismo material activo. La técnica de deposición demuestra que se pueden obtener dispositivos con alta reproducibilidad y que en todos los casos muestran una baja tensión umbral de alrededor 1 V. Por lo tanto, se demuestra que la deposición convectiva rápida puede ser usada para la fabricación de transistores orgánicos de efecto de campo sobre áreas amplias, con indicadores de reproducibilidad entre dispositivos y alta estabilidad en condiciones ambiente.

Palabras clave: transistores orgánicos de efecto de campo, deposición convectiva rápida, materiales compuestos

\section{Abstract}

Organic field-effect transistors based on composite materials has been manufactured using the rapid convective deposition technique. The manufacturing was carried out under environmental conditions (air, light and humidity). All manufactured transistors show a typical field-effect behavior with features of a p-type semiconductor, and exhibit field-effect mobilities around $10^{-2} \mathrm{~cm}^{2} / \mathrm{V} . \mathrm{s}$, fully comparable with transistors manufactured using thermal evaporation of the same active material. The deposition technique demonstrates that devices may be manufactured with high reproducibility and in all cases with a low threshold voltage of approximately $1 \mathrm{~V}$. Therefore, it is demonstrated here that rapid convective deposition can be used to manufacture organic fieldeffect transistors on large surface areas, showing high reproducibility among devices and high stability at environmental conditions.

Keywords: Organic field-effect transistors, Rapid convective deposition, Composite Materials.

\footnotetext{
1,* Facultad de Ciencia e Ingeniería en Alimentos y Biotecnología, Universidad Técnica de Ambato - Ecuador. Autor para correspondencia : fg.delpozo@uta.edu.ec.

${ }^{2}$ Departamento de Nanociencia Molecular y Materiales Orgánicos, Instituto de Ciencia de Materiales de Barcelona (ICMAB - CSIC)/ CIBER - BBN, 08193 - Bellaterra - España.
}

Forma sugerida de citación: Del Pozo, F. G. y Mas-Torrent, M. (2021). «Deposición convectiva rápida a escala nanométrica de materiales compuestos activos para la fabricación de transistores orgánicos de efecto de campo». InGENIUS. N. ${ }^{\circ}$ 26, (julio-diciembre). pp. 9-16. DOI: https://doi.org/10.17163/ings.n26.2021.01. 


\section{Introducción}

El desarrollo de la electrónica orgánica, particularmente en las dos décadas pasadas, ha sido de vital importancia y ha producido grandes impactos en la comunidad de investigadores y en la industria electrónica. Algunos de los dispositivos con arquitectura orgánica, como los diodos orgánicos emisores de luz (OLED), hoy por hoy brillan con un gran éxito y en el momento están siendo producidos en serie. Así, es fácil ya encontrar en nuestra vida diaria dispositivos como televisores o celulares con pantallas basadas en OLED $[1,2]$.

Actualmente, los semiconductores han sido adoptados en un amplísimo rango de aplicaciones como pantallas, computadores, teléfonos, entre otros, volviéndose indispensables en nuestra vida cotidiana, y casi sin habernos dado cuenta.

Los núcleos de todos los dispositivos orgánicos son los semiconductores orgánicos (OS) y estos pueden ser usados como material activo de diversos dispositivos desde dispositivos unitarios (transistores, diodos, sensores) desarrollados en laboratorios de investigación, hasta circuitos complejos. El descubrimiento de los semiconductores orgánicos data ya de los años 1940 [3]. En el tenor de comparar con la electrónica tradicional basada en el silicio, la electrónica orgánica muestra características únicas, como procesamiento a partir de soluciones, flexibilidad mecánica e incluso una mezcla de conducción iónica y eléctrica, lo que hace que la electrónica orgánica haya captado el interés de muchos laboratorios de investigación en todo el mundo.

Los OS están caracterizados por ser moléculas conjugadas o incluso polímeros conjugados. Su estructura difiere enormemente de la de los tradicionales semiconductores inorgánicos, ya que en los OS son característicos los enlaces débiles de Van der Waals, mientras que en los semiconductores inorgánicos los enlaces covalentes prevalecen, además, los electrones $\pi$ de los OS forman las bandas de valencia y de conducción. Algo muy importante de resaltar es que los OS, a diferencia de los semiconductores inorgánicos, no forman una capa de óxido nativo, por ende propician una interfaz limpia entre los materiales, ya que los materiales que pueden interactuar con los OS varían desde metales hasta organismos biológicos [4].

Los esfuerzos en investigación se han enfocado en la manipulación de las estructuras químicas de diversos OS, su grado de ordenamiento en capas nanométricas, como en el comprender y controlar los procesos de transporte que se desarrollan en las interfaces [5]. Sin embargo, con la finalidad de poder desarrollar dispositivos de bajo costo, es necesario el procesamiento de los OS con técnicas basadas en disoluciones de estos, para llegar a lo que se conoce como electrónica impresa.

Los semiconductores poliméricos gozan de una buena solubilidad en una amplia gama de disolventes orgánicos, sin embargo, los semiconductores de moléculas pequeñas muestran problemas en su solubilidad [2].

El principio de operación de un transistor orgánico de efecto de campo (OFET) se basa en la aplicación de un campo eléctrico, el mismo que causa la formación de un canal de conducción en la interfaz del dieléctrico y el semiconductor orgánico. Las configuraciones típicas y más usadas son las que se muestran en la Figura 1 y se las conoce como de contactos superiores y de contactos inferiores.

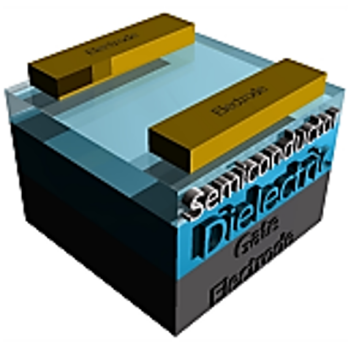

(a)

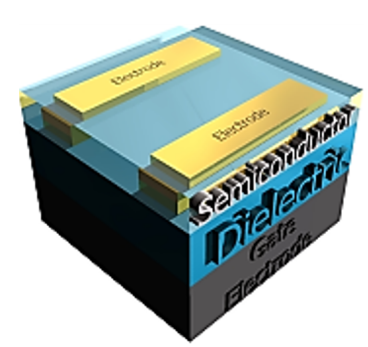

(b)
Figura 1. Configuraciones típicas usadas en la práctica de OFET: (a) contactos superiores, (b) contactos inferiores

En ambos casos, el semiconductor orgánico que se deposita en una capa de un dieléctrico está en contacto con dos electrodos metálicos, denominados fuente y sumidero; y en la parte inferior el electrodo de puerta es típicamente colocado. Entonces, la corriente que fluye entre la fuente y el sumidero $\left(I_{S D}\right)$ puede ser modulada por el voltaje aplicado en el electrodo de la puerta $\left(V_{G}\right)$ entre la fuente y la puerta. Este voltaje desarrolla un campo eléctrico y es él el responsable de la formación de una capa de acumulación de cargas en la interfaz semiconductora/dieléctrica. Además, dependiendo de la direccionalidad del voltaje aplicado en la puerta $\left(V_{G}\right)$, la naturaleza de los portadores de carga que se acumulan en la interfaz pueden ser controlados, así, huecos para semiconductores tipo-p y electrones para semiconductores tipo-n [6].

Los OS pueden ser depositados sobre superficies en fase de vapor o en solución, los procesos son dependientes de la presión de vapor y de la solubilidad del material semiconductor. Los distintos métodos de deposición derivan en distintas morfologías de las películas resultantes, como también incrementan la variabilidad en el desempeño de los dispositivos elaborados. Sin embargo, durante los años pasados nuevos OS funcionalizados han emergido, se han añadido grupos funcionales que han mejorado la solubilidad de los núcleos conjugados rígidos. Esto ha permitido mejorar drásticamente la solubilidad sin perder las propiedades eléctricas inherentes, ni sacrificar su empaquetamiento molecular [2], [5].

De esta manera, un factor muy importante a considerar es la fabricación de capas a escala nanométrica que permita el depósito de OS funcionalizados o no, 
sobre sustratos que posteriormente permitan la construcción de arquitecturas de transistores. Uno de los OS más prometedores que se han encontrado es el dibenzotetratiafulvaleno (DB-TTF), por ello fue seleccionado para este estudio [7-10]. Desde el punto de vista de los procesos de fabricación, las películas delgadas orgánicas han sido manufacturadas con procesos a bajas temperaturas, entre los cuales destaca el recubrimiento por centrifugación que es ampliamente usado en diversos laboratorios de investigación. El recubrimiento por centrifugación permite tener control sobre el espesor de las películas fabricadas que en su gran mayoría son homogéneas y sin rupturas; sin embargo, dicho método de procesamiento no puede ser escalado.

Lo que hace que el recubrimiento por centrifugación no se pueda usar en superficies grandes de procesamiento, debido a la necesidad de usar grandes cantidades de solución, lo que hace que el método de recubrimiento por centrifugación no sea viable para su aplicación en la fabricación de transistores orgánicos de efecto de campo fabricados sobre áreas extensas. Por esta razón, el desarrollo de técnicas de fabricación que reduzcan el desperdicio de la solución del OS, que típicamente es costosa, y que de igual manera la nueva técnica de fabricación permita el depósito de capas delgadas de materiales orgánicos sobre sustratos, es un interés de investigación constante. Dentro de los métodos poco convencionales, el autoensamblaje por convección es uno ampliamente usado para el depósito de recubrimientos sobre áreas extensas en una escala de tiempo pequeña.

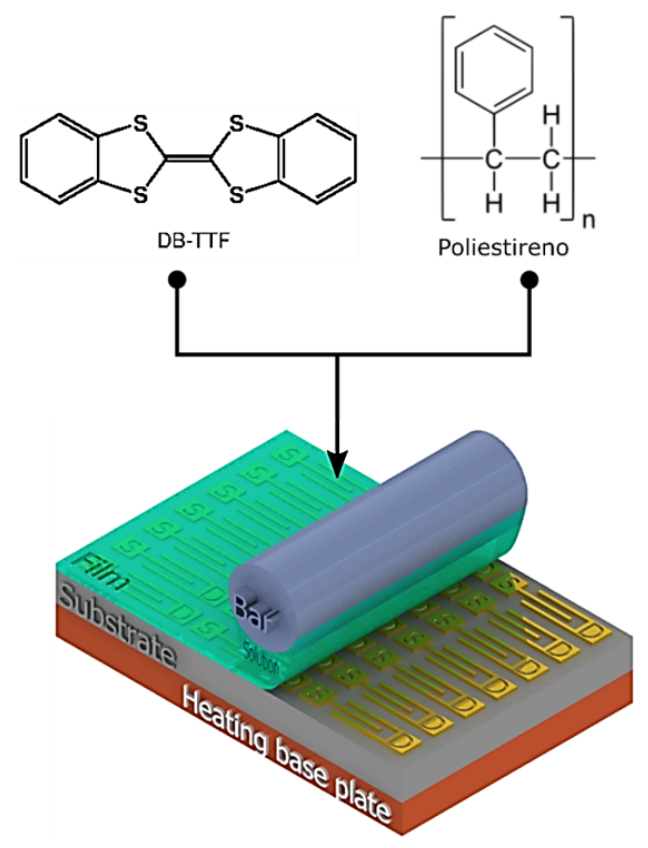

Figura 2. Esquema gráfico típico de la deposición convectiva rápida de polímeros aislantes, semiconductores y/o de sus mezclas
Una forma de usar el autoensamblaje por convección, o también conocido como deposición convectiva rápida, es usar un recubridor de barra como el que se muestra en la Figura 2 para dispersar la solución del OS sobre el sustrato deseado y provocando el autoensamblaje de las moléculas seleccionadas sobre la superficie del sustrato [11], [12].

A pesar de las prometedoras características de los semiconductores tipo molécula pequeña, la fabricación de filmes homogéneos es complicada, sin embargo, para poder evadir esa dificultad, la ruta más prometedora hasta el día de hoy es el conformar materiales compuestos, en mezclas típicas de materiales poliméricos como matriz y como material activo el semiconductor orgánico. La aplicación de materiales compuestos ha facilitado enormemente la procesabilidad de los semiconductores orgánicos y, además, han mejorado la cristalinidad de las películas obtenidas, como también han mejorado la estabilidad de las películas frente a condiciones ambientales [2], [13].

\section{Materiales y métodos}

Los sustratos de silicio fueron adquiridos a Fraunhofer IPMS y tienen un dopaje tipo n, además de una capa de dióxido de silicio de $230 \mathrm{~nm}$ de espesor. Los electrodos de oro tienen una altura de $30 \mathrm{~nm}$, y fueron depositados sobre una capa de óxido delgado de indio (ITO) de $10 \mathrm{~nm}$ de espesor, que actúa como una capa de adhesión. Las películas térmicamente evaporadas de dibenzo tetratiafulvaleno (DB-TTF) fueron preparadas a una velocidad lenta de evaporación de alrededor de $0,5 \AA /$ s sobre sustratos de $\mathrm{Si} / \mathrm{SiO}_{2}$ con contactos de ITO/Au, el espesor de las películas se determinó de $110 \mathrm{~nm}$ por medio de un análisis de AFM. Las películas delgadas del material compuesto activo fueron producidas al adaptar un recubridor comercial a las necesidades del experimento. DB-TTF y poliestireno para GPC Mw 10000 (PS10k) fueron adquiridos de Sigma-Aldrich y usados sin purificación subsecuente alguna.

En este estudio se usa poliestireno para GPC Mw 10000 (PS10k) junto con el semiconductor orgánico DB-TTF. Se prepararon soluciones al $2 \%$ en clorobenzeno anhidro tanto del polímero aislante como del semiconductor. Asimismo, usando la proporción 1:3 se preparó solución de semiconductor versus solución del polímero aislante en viales separados que, en todos los casos, las mezclas no superan el volumen de $500 \mu l$. Silicio altamente dopado de tipo-n, y con un espesor de $200 \mathrm{~nm}$ de óxido de silicio nativo (dieléctrico) se usaron como sustrato y electrodo de puerta, respectivamente. Los sustratos empleados para los recubrimientos se adquirieron a Si-mat, en los cuales usando fotolitografía se diseñaron los electrodos que consisten en una capa de $4 \mathrm{~nm}$ de $\mathrm{Cr}$ (capa de adhe- 
sión) y $40 \mathrm{~nm}$ de $\mathrm{Au}$, ambos metales depositados por evaporación térmica. Los sustratos, previo a ingresar al proceso de recubrimiento, son limpiados con acetona e isopropanol, ambos disolventes de calidad HPLC. Finalmente, los sustratos son secados bajo una corriente de nitrógeno.

El equipo de recubrimiento fue precalentado a $105^{\circ} \mathrm{C}$ antes de los experimentos. Los sustratos con electrodos prefabricados fueron limpiados previamente. Los sustratos tienen que ser dispuestos con cuidado en la máquina de recubrimiento; para realizar el mismo se usa una pequeña cantidad de solución correspondiente que oscila por 30 pl. La solución es depositada, y el menisco arrastrado a una velocidad constante de $10 \mathrm{~mm} / \mathrm{s}$; durante el proceso de deposición convectiva rápida, siempre el sustrato es mantenido estático y a $105{ }^{\circ} \mathrm{C}$. Inmediatamente después del proceso de recubrimiento, los sustratos recubiertos son cuidadosamente removidos del equipo y dispuestos en cajas Petri, y luego sometidos a una etapa de curado en vacío $\left(P_{a b s}=7 \mathrm{kPa}\right)$ y a $60{ }^{\circ} \mathrm{C}$ por al menos un periodo de $2 \mathrm{~h}$, con la finalidad de remover cualquier vestigio de disolvente. Luego del curado, las características de los transistores son medidas a temperatura ambiente y en oscuridad.

Las fotografías de microscopía óptica fueron obtenidas usando un microscopio Olympus BX51 equipado con polarizador y analizador. El estudio de la sección transversal de las películas se realizó utilizando una estación de trabajo FIB-SEM Neon40 Crossbeam $^{\mathrm{TM}}$ de Carl Zeiss. La topografía de la superficie de las películas depositadas fue estudiada gracias al uso de un SPM 5500LS de Agilent Technologies; el tratamiento de datos de topografía se realizó con el paquete informático Gwyddion. Las medidas de difracción de rayos $\mathrm{X}$ fueron realizadas con un difractómetro de Rigaku, equipado con un ánodo de fuente rotacional.

Las medidas eléctricas se realizaron con un Keithley $2612 \mathrm{AB}$ con una interfaz programada en MATLAB®, típicamente en una estación de pruebas Süss con sondas tipo punta y en oscuridad.

\section{Resultados y discusión}

La Figura 3 muestra una imagen obtenida por microscopía óptica de una película de DB-TTF:PS10 k en una razón de mezcla de 1:3 depositada en una arquitectura de contactos inferiores; se puede observar una película bien formada y libre de rupturas sobre toda el área fotografiada. Es importante el notar que, a pesar de ser una arquitectura de contactos inferiores, la película depositada es de carácter homogéneo.

Ya que una de las principales características de la molécula pequeña y semiconductora de DB-TTF es la cristalinidad, la Figura 4 presenta la imagen por microscopía óptica polarizada en la cual se muestra y, claramente, se puede inferir las estructuras cristalinas (eferulitas) formadas; estas están embebidas en la película depositada por deposición convectiva rápida, y las redes cristalinas formadas junto con vías también cristalinas para el transporte de huecos entre los electrodos.

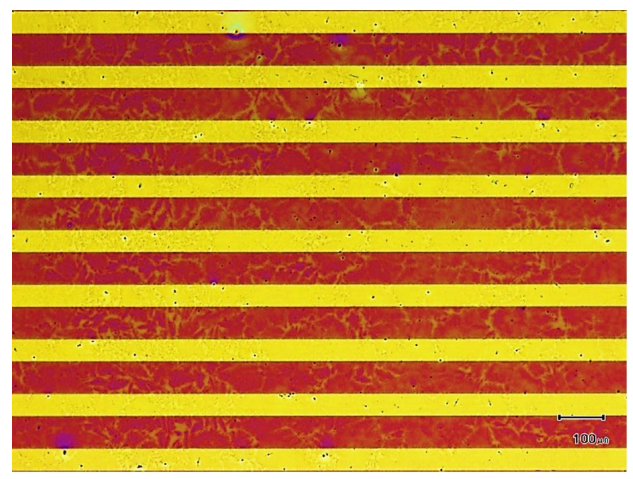

Figura 3. Fotografía obtenida por microscopía óptica de una película típica del material compuesto DB-TTF y PS10k en una relación 1:3 el ángulo entre el polarizador y el analizar es de $0^{\circ}$

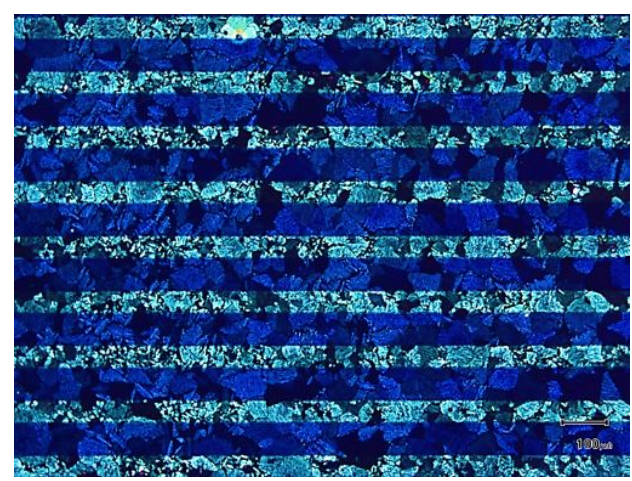

Figura 4. Fotografía obtenida por microscopía óptica de una película típica del material compuesto DB-TTF y PS10k en una relación 1:3 el ángulo entre el polarizador y el analizar es de $90^{\circ}$

Cuando se usan materiales compuestos, los parámetros como la naturaleza del polímero, la relación entre el polímero y el material activo, combinados con los parámetros mecánicos del proceso de deposición vuelven a los sistemas complejos. En el campo de los semiconductores orgánicos, todas las fases cristalinas de los OS son obtenidas a pesar de que no lleguen al equilibrio termodinámico, así aquellas fases obtenidas deben modificar los parámetros extrínsecos. Estas fases cristalinas observadas serán nombradas en función de los polimorfos cinéticos. A pesar de que el DB-TTF muestra polimorfismo complejo se logra obtener una fase cinética cristalina, y en la Figura 4 se observa la fase cinética gamma que tiene formas tipo placa, las mismas son observables con facilidad a lo largo de los electrodos del dispositivo analizado. Debido a que 
la técnica de deposición promueve una cristalización rápida, la misma tiende a formar la fase cristalina cinética más favorable [14-16].

Las Figuras 5 y 6 muestran los fotogramas obtenidos por FIB-SEM, técnica que es ampliamente usada para el estudio de secciones transversales de dispositivos y que, de igual manera, permite el estudio de las interfaces entre materiales, gracias a un haz de iones de Ga+ enfocado que es usado para la obtención de la imagen e incluso empleado para realizar nanoestructuración. Con la finalidad de calibrar y obtener un corte limpio, se evaporan $100 \mathrm{~nm}$ de Pt sobre la muestra, esto se observa en la Figura 5, juntamente con el agujero realizado.

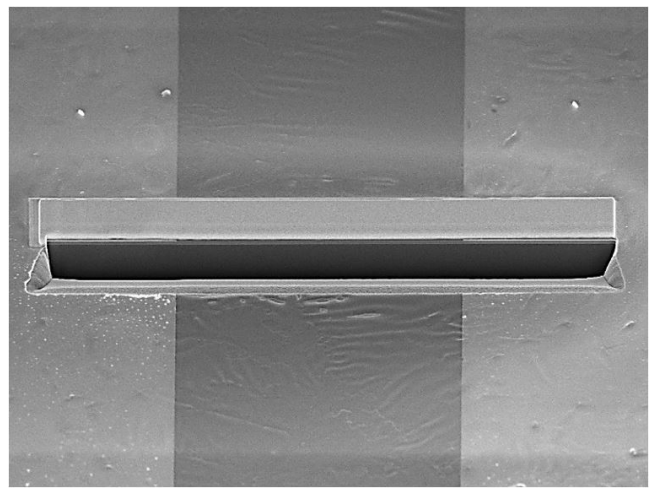

Figura 5. Imagen de FIB-SEM de un dispositivo típico fabricado de una película del material compuesto DBTTF y PS10k en una relación 1:3. Mag $=6.89 \mathrm{kX}$, $\mathrm{EHT}=5.00 \mathrm{kV}$, FIB Probe $=30 \mathrm{kV}: 10 \mathrm{pA}$, FIB Imaging $=$ SEM, vacuum $=1.72 \mathrm{e}-6$ mbar

Mientras que la Figura 6. muestra la sección transversal de la muestra, posterior a la rotación al interno del FIB-SEM que, además, se nota el Pt, el $\mathrm{SiO}_{\mathrm{x}}$, Si. Gracias a esta técnica se puede medir un espesor de 52,33 nm de la película depositada sobre el sustrato con contactos inferiores.

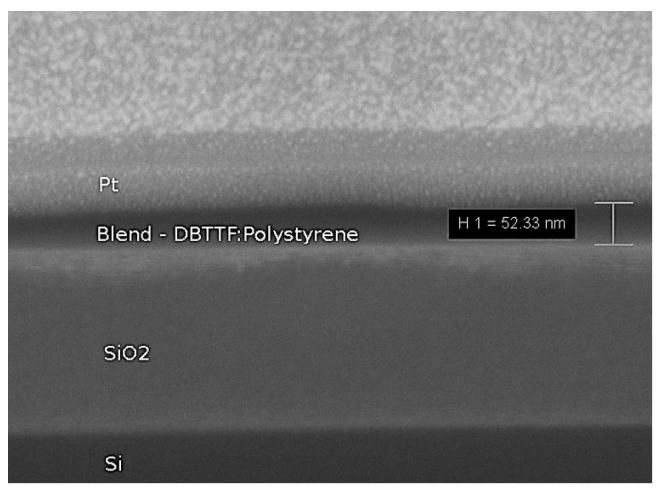

Figura 6. Imagen de FIB-SEM de la sección transversal de un dispositivo típico fabricado de una película del material compuesto DB-TTF y PS10k en una relación 1:3 $\mathrm{Mag}=6,89 \mathrm{kX}, \mathrm{EHT}=5,00 \mathrm{kV}$, FIB Probe $=30 \mathrm{kV}: 10 \mathrm{pA}$, FIB Imaging $=$ SEM, vacuum $=1,72 \mathrm{e}-6$ mbar
La Figura 7 fue obtenida por AFM, en la que se muestran estructuras no periódicas y no estructuradas en una región en particular de la película depositada. Además, se observa una variabilidad en su topografía con un máximo de 41,6 nm.

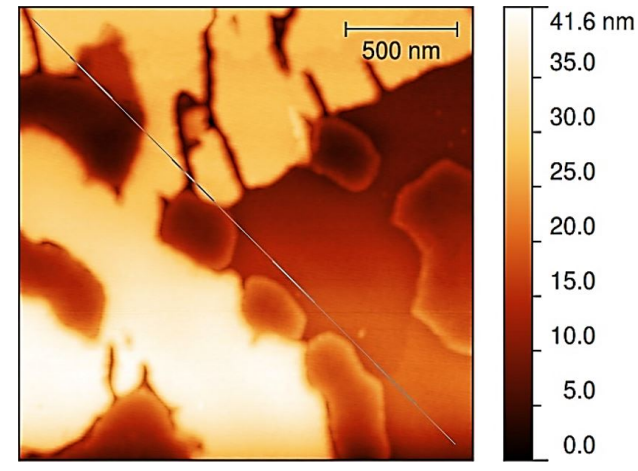

Figura 7. Imagen de AFM de un dispositivo típico fabricado de una película del material compuesto DB-TTF y PS10k en una relación 1:3

La Figura 8 muestra la rugosidad en función de la posición, y es obtenida a lo largo del perfil diagonal que se muestra en la Figura 7, así la película depositada muestra una rugosidad relativamente baja con un máximo de 2,0 $\mathrm{nm}$ en la posición aproximada de $1 \mu m$ positiva en el eje X. La superficie lisa es un indicativo de poder tener una interfaz limpia con el potencial en el futuro a ser usada para otras aplicaciones, como transistores orgánicos de efecto de campo con electrodos-puerta electrolíticos e incluso en el desarrollo de sensores [17].

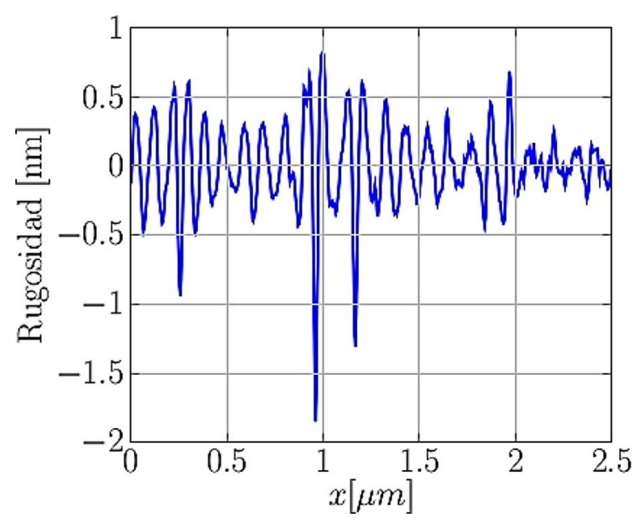

Figura 8. Rugosidad (nm) en función de la posición en el eje $\mathrm{X}[\mu \mathrm{m}]$ extraída del perfil diagonal mostrado en la Figura 7, para un dispositivo típico fabricado de una película del material compuesto DB-TTF y PS10k en una relación 1:3

Debido a la naturaleza cristalina del material activo DB-TTF el estudio por difracción de rayos X es indispensable, ya que el conocimiento de la estructura cristalina es clave para poder determinar y comprender las características eléctricas de un material. La 
Figura 9 muestra el difractograma, tanto de la película obtenida con la deposición convectiva rápida como la obtenida con el material puro DB-TTF evaporado térmicamente con la finalidad de comprobar la formación de redes cristalinas, y descartar que se obtenga una película amorfa.

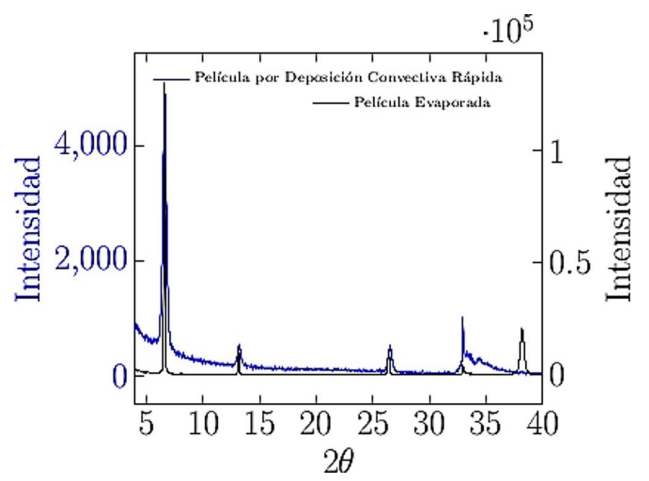

Figura 9. Difractograma de rayos $X$ para una película térmicamente evaporada de DB-TTF, y de una película típica del material compuesto DB-TTF y PS10k en una relación 1:3

Entonces, la Figura 9 muestra las coincidencias en los picos, los se observan a $6,72^{\circ}, 13,48^{\circ}, 20,22^{\circ}$, $27,08^{\circ}$ y $41,22^{\circ}$. Así se puede adscribir a la formación de la fase cristalina gamma según lo reportado por Brillante y colaboradores [18], sin embargo, la estructura cristalina de la fase gamma aún no ha sido resuelta. Notablemente, la celda unidad de la fase gamma contiene dos moléculas de DB-TTF, a pesar de que el incremento de volumen por molécula en esta fase versus el polimorfo-alfa indica que el polimorfo-gamma es una fase menos estable [14].

Las Figuras 10 y 11 muestran las características de salida y de transferencia para un dispositivo típico basado en el material compuesto activo DBTTF:PS10k (1:3). La imagen de mérito mostrada en Figura 10 en su característica de salida muestra características que son reproducibles entre dispositivos y que muestran un comportamiento casi-ideal en su respuesta lineal y de saturación.

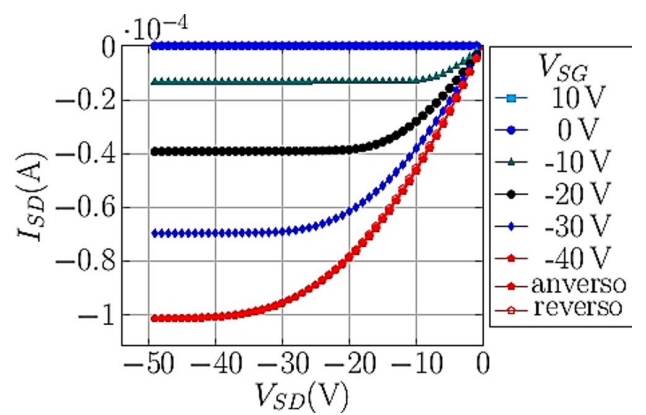

Figura 10. Característica de salida del OFET de una película típica del material compuesto DB-TTF y PS10k en una relación 1:3. Dispositivo $\mathrm{L}=75 \mathrm{um}$, $\mathrm{W}=75000 \mathrm{um}$

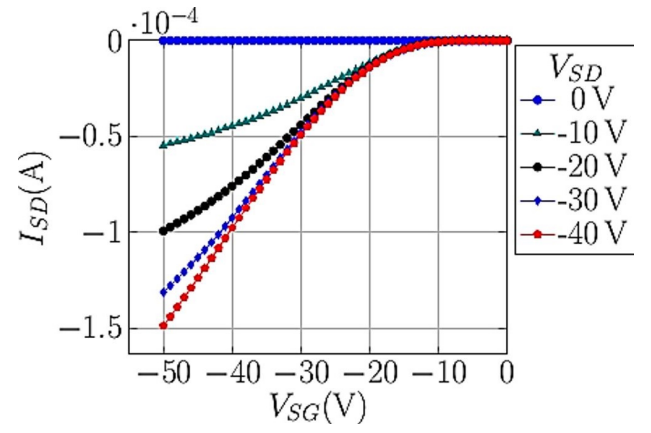

Figura 11. Característica de transferencia de una película típica del material compuesto DB-TTF y PS10k en una relación 1:3. Dispositivo $\mathrm{L}=75 \mathrm{um}, \mathrm{W}=75000 \mathrm{um}$

Entonces, el dispositivo analizado eléctricamente muestra características típicas de efecto de campo, observadas en la Figura 10 de la característica de salida, en la cual es claro que el voltaje aplicado en la puerta modula la corriente medida entre los electrodos fuente y sumidero; de igual manera, se observa cómo la corriente fuente-sumidero tiende a saturar a medida que se acerca al voltaje aplicado en el electrodo de puerta. También es importante mencionar la mínima histéresis formada durante el mapeo de corrientes-voltajes en dirección positiva (barrido anverso) y en dirección opuesta (barrido reverso). Una característica distintiva de la existencia de trampas es la histéresis del dispositivo, donde existe una diferencia significativa entre los barridos anverso y reverso, esta puede ocurrir cuando las cargas se ven aprisionadas y luego liberadas, respectivamente y en conjunto puede darse debido a dieléctricos muy polares [5].

La Tabla 1 resume los valores de movilidad y de tensión umbral extraídas en el régimen de saturación para varias longitudes de canal; a pesar de la diferencia significativa en la longitud de canal, la movilidad permanece entre los mismos valores, sugiriendo la homogeneidad de fabricación entre distintos dispositivos seleccionados. La tensión umbral da a claras que los transistores necesitan muy poco voltaje para encenderse, y la característica de transferencia mostrada en la Figura 11 sugiere que existen al menos cuatro órdenes de magnitud entre el estado apagado y el estado encendido en los transistores orgánicos de efecto de campo fabricados.

Ya que los OFET son considerados y visionados para ser elementos en aplicaciones tecnológicas, la fabricación a bajo costo y la estabilidad al ambiente son factores cruciales a considerar [19]. De esta manera, y con la finalidad de probar la estabilidad en el tiempo bajo condiciones controladas, un transistor fue sometido a diversas mediciones al interior de una incubadora $\left(\mathrm{T}=25^{\circ} \mathrm{C}\right)$. Así, la Figura 12 muestra la característica de transferencia logarítmica medida sobre espacios de tiempo discretos; dicha gráfica sugiere que la estabilidad ha sido mejorada significativamente, 
ya que filmes de DB-TTF evaporados térmicamente son extremadamente sensibles a condiciones ambientales [12], [20], [7].

Tabla 1. Resumen de datos de la movilidad y tensión umbral en función de la longitud del canal del transistor

\begin{tabular}{ccc}
\hline $\begin{array}{c}\text { Longitud de } \\
\text { canal }[\mu \mathrm{m}]\end{array}$ & $\begin{array}{c}\text { Movilidad } \\
{\left[\mathrm{cm}^{2} / \mathrm{V} . \mathrm{s}\right]}\end{array}$ & $\begin{array}{c}\text { Tensión } \\
\text { umbral }[\mathrm{V}]\end{array}$ \\
\hline 25 & $9,75 \mathrm{e}-3$ & $-1,81$ \\
50 & $1,42 \mathrm{e}-2$ & 0,38 \\
75 & $1,02 \mathrm{e}-2$ & 1,18 \\
10 & $1,27 \mathrm{e}-2$ & $-1,11$ \\
\hline
\end{tabular}

Nota. Todos los dispositivos son fabricados a partir de una película típica del material compuesto DB-TTF y PS10k en una relación $1: 3$.

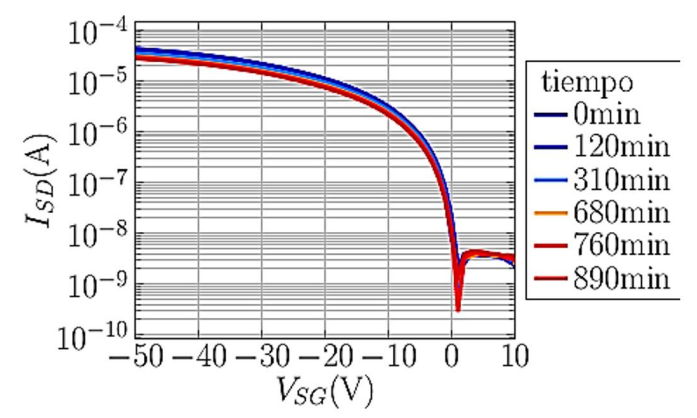

Figura 12. Característica de transferencia logarítmica para un dispositivo fabricado de una película típica del material compuesto DB-TTF y PS10k en una relación 1:3

\section{Conclusiones}

Transistores orgánicos de efecto de campo a partir de un material compuesto basado en un TTF como material activo, han sido satisfactoriamente fabricados a partir de una metodología basada en la deposición convectiva rápida a partir de una disolución del material compuesto. Para lograr estabilidad ambiental se escogió el poliestireno como matriz ligante. Todos los dispositivos fueron fabricados y medidos en condiciones ambientales de luz y humedad, exhibiendo una mejora drástica en su estabilidad en comparación con los fabricados por evaporación térmica. Las movilidades de los dispositivos fabricados oscilan el $10^{-2} \mathrm{~cm}^{2} /$ V.s con un $\pm 10^{-3} \mathrm{~cm}^{2} /$ V.s, dicha movilidad es perfectamente comparable con los dispositivos fabricados por evaporación térmica a partir de DB-TTF puro. Por lo tanto, se demuestra que la técnica de fabricación aplicada a un material compuesto provee una ruta prometedora para la fabricación de dispositivos de bajo costo y con alta estabilidad.

\section{Agradecimientos}

Freddy del Pozo quiere agradecer al Grupo NANOMOL del Instituto de Ciencia de Materiales de Barcelona (CSIC) por haberme acogido durante la formación doctoral. También a la Secretaría de Educación Superior, Ciencia, Tecnología e Innovación (Senescyt) por el financiamiento otorgado por medio de la beca de doctorado. A la Universidad Técnica de Ambato y a sus autoridades. Agradecimiento a la Dirección de Investigación y Desarrollo (DIDE) de la Universidad Técnica de Ambato por el proyecto «Deposición de capas orgánicas semiconductoras a escala nanométrica para la fabricación de transistores orgánicos de efecto de campo» aprobado con resolución 0911-CU-P-2016. Al doctor Puigdollers y al Centro de Nanoestructuración de la Universidad Politécnica de Catalunya.

\section{Referencias}

[1] P. K. L. Chan, "The motivation for and challenges to scaling down organic field-effect transistors," Advanced Electronic Materials, vol. 5, no. 7, p. 1900029, 2019. [Online]. Available: https://doi.org/10.1002/aelm.201900029

[2] S. Riera-Galindo, F. Leonardi, R. Pfattner, and M. Mas-Torrent, "Organic semiconductor/polymer blend films for organic field-effect transistors," Advanced Materials Technologies, vol. 4, no. 9, p. 1900104, 2019. [Online]. Available: https://doi.org/10.1002/admt.201900104

[3] C. Wang, H. Dong, L. Jiang, and W. Hu, "Organic semiconductor crystals," Chemical Society Reviews, vol. 47, pp. 422-500, 2018. [Online]. Available: http://dx.doi.org/10.1039/C7CS00490G

[4] M. Fahlman, S. Fabiano, V. Gueskine, D. Simon, M. Berggren, and X. Crispin, "Interfaces in organic electronics," Nature Reviews Materials, vol. 4, no. 10, pp. 627-650, Oct. 2019. [Online]. Available: https://doi.org/10.1038/s41578-019-0127-y

[5] Z. A. Lamport, H. F. Haneef, S. Anand, M. Waldrip, and O. D. Jurchescu, "Tutorial: Organic field-effect transistors: Materials, structure and operation," Journal of Applied Physics, vol. 124, no. 7, p. 071101, 2018. [Online]. Available: https://doi.org/10.1063/1.5042255

[6] M. Mas-Torrent and C. Rovira, "Role of molecular order and solid-state structure in organic field-effect transistors," Chemical Reviews, vol. 111, no. 8, pp. 4833-4856, 2011, pMID: 21417271. [Online]. Available: https://doi.org/10.1021/cr100142w 
[7] K. Shibata, K. Ishikawa, H. Takezoe, H. Wada, and T. Mori, "Contact resistance of dibenzotetrathiafulvalene-based organic transistors with metal and organic electrodes," Applied Physics Letters, vol. 92, no. 2, p. 023305, 2008. [Online]. Available: https://doi.org/10.1063/1.2834374

[8] B. Noda, H. Wada, K. Shibata, T. Yoshino, M. Katsuhara, I. Aoyagi, T. Mori, T. Taguchi, T. Kambayashi, K. Ishikawa, and H. Takezoe, "Crystal structures and transistor properties of phenyl-substituted tetrathiafulvalene derivatives," Nanotechnology, vol. 18, no. 42, p. 424009, sep 2007. [Online]. Available: https: //doi.org/10.1088/0957-4484/18/42/424009

[9] M. Mas-Torrent, P. Hadley, S. T. Bromley, X. Ribas, J. Tarrés, M. Mas, E. Molins, J. Veciana, and C. Rovira, "Correlation between crystal structure and mobility in organic fieldeffect transistors based on single crystals of tetrathiafulvalene derivatives," Journal of the American Chemical Society, vol. 126, no. 27, pp. 8546-8553, 2004, pMID: 15238013. [Online]. Available: https://doi.org/10.1021/ja048342i

[10] M. Mas-Torrent, P. Hadley, S. T. Bromley, N. Crivillers, J. Veciana, and C. Rovira, "Singlecrystal organic field-effect transistors based on dibenzo-tetrathiafulvalene," Applied Physics Letters, vol. 86, no. 1, p. 012110, 2005. [Online]. Available: https://doi.org/10.1063/1.1848179

[11] T. Chonsut, A. Rangkasikorn, S. Wirunchit, A. Kaewprajak, P. Kumnorkaew, N. Kayunkid, and J. Nukeaw, "Rapid convective deposition; an alternative method to prepare organic thin film in scale of nanometer," Materials Today: Proceedings, vol. 4, no. 5, Part 2, pp. 6134-6139, 2017, international Conference on Science and Technology of the Emerging Materials (July 27-29, 2016), Pattaya, Thailand. [Online]. Available: https://doi.org/10.1016/j.matpr.2017.06.106

[12] F. G. D. Pozo, S. Galindo, R. Pfattner, C. Rovira, and M. Mas-Torrent, "Deposition of composite materials using a wire-bar coater for achieving processability and air-stability in Organic Field-Effect Transistors (OFETs)," in Organic Field-Effect Transistors XIV; and Organic Sensors and Bioelectronics VIII, I. Kymissis, R. Shinar, L. Torsi, I. McCulloch, and O. D. Jurchescu, Eds., vol. 9568, International Society for Optics and Photonics. SPIE, 2015, pp. 17-22. [Online]. Available: https://doi.org/10.1117/12.2186521

[13] L. J. Richter, D. M. DeLongchamp, and A. Amassian, "Morphology development in solution-processed functional organic blend films: An in situ viewpoint," Chemical Reviews, vol. 117, no. 9, pp. 6332-6366, 2017, pMID: 28414244. [Online]. Available: https://doi.org/10.1021/acs.chemrev.6b00618

[14] A. Tamayo, S. Riera-Galindo, A. O. F. Jones, R. Resel, and M. Mas-Torrent, "Impact of the ink formulation and coating speed on the polymorphism and morphology of a solution-sheared thin film of a blended organic semiconductor," Advanced Materials Interfaces, vol. 6, no. 22 , p. 1900950, 2019. [Online]. Available: https://doi.org/10.1002/admi.201900950

[15] A. M. Hiszpanski, R. M. Baur, B. Kim, N. J. Tremblay, C. Nuckolls, A. R. Woll, and Y.-L. Loo, "Tuning polymorphism and orientation in organic semiconductor thin films via postdeposition processing," Journal of the American Chemical Society, vol. 136, no. 44, pp. 15749 15 756, 2014, pMID: 25317987. [Online]. Available: https://doi.org/10.1021/ja5091035

[16] H. Chung and Y. Diao, "Polymorphism as an emerging design strategy for high performance organic electronics," Journal of Materials Chemistry $C$, vol. 4, pp. 3915-3933, 2016. [Online]. Available: http://dx.doi.org/10.1039/C5TC04390E

[17] A. Kyndiah, F. Leonardi, C. Tarantino, T. Cramer, R. Millan-Solsona, E. Garreta, N. Montserrat, M. Mas-Torrent, and G. Gomila, "Bioelectronic recordings of cardiomyocytes with accumulation mode electrolyte gated organic field effect transistors," Biosensors and Bioelectronics, vol. 150, p. 111844, 2020. [Online]. Available: https://doi.org/10.1016/j.bios.2019.111844

[18] A. Brillante, I. Bilotti, R. G. Della Valle, E. Venuti, S. Milita, C. Dionigi, F. Borgatti, A. N. Lazar, F. Biscarini, M. Mas-Torrent, N. S. Oxtoby, N. Crivillers, J. Veciana, C. Rovira, M. Leufgen, G. Schmidt, and L. W. Molenkamp, "The four polymorphic modifications of the semiconductor dibenzo-tetrathiafulvalene," CrystEngComm, vol. 10, pp. 1899-1909, 2008. [Online]. Available: http://dx.doi.org/10.1039/B810993A

[19] P. A. Bobbert, A. Sharma, S. G. J. Mathijssen, M. Kemerink, and D. M. de Leeuw, "Operational stability of organic field-effect transistors," Advanced Materials, vol. 24, no. 9, pp. 1146-1158, 2012. [Online]. Available: https://doi.org/10.1002/adma.201104580

[20] M. Mas-Torrent and C. Rovira, "Tetrathiafulvalene derivatives for organic field effect transistors," Journal of Materials Chemistry, vol. 16, pp. 433-436, 2006. [Online]. Available: http://dx.doi.org/10.1039/B510121B 